\title{
Implications for biological function of lobe dependence of the molecular structure of liver glycogen
}

\author{
Zhenxia Hu, ${ }^{\mathrm{a}, \mathrm{b}}$ Xinle Tan, ${ }^{\mathrm{c}}$ Bin Deng, ${ }^{\mathrm{d}}$ Hua Gan, ${ }^{\mathrm{a}}$ Xiaoyin Jiang, ${ }^{\mathrm{a}}$ Kai \\ Wang, ${ }^{\mathrm{e}}$ Cheng Li, ${ }^{\mathrm{b}}$ Enpeng Li, ${ }^{\mathrm{b} *}$ and Robert G. Gilbert ${ }^{\mathrm{b}, \mathrm{c}^{*}}$
}

${ }^{a}$ School of Pharmacy, Tongji Medical College, Huazhong University of Science and Technology, Wuhan, Hubei, China, 430030

${ }^{b}$ Joint International Research Laboratory of Agriculture and Agri-Product Safety, College of Agriculture, Yangzhou University, Yangzhou 225009, Jiangsu Province, China

${ }^{c}$ The University of Queensland, Centre for Nutrition and Food Science, Queensland Alliance for Agriculture and Food Innovation, Brisbane, QLD 4072, Australia

${ }^{d}$ Department of Pharmacy, Union Hospital, Tongji Medical College, Huazhong University of Science and Technology, Wuhan, Hubei 430030, China

${ }^{e}$ College of Food Science, South China Agricultural University, Guangzhou, Guangdong 510642, China

* Corresponding authors at: Joint International Research Laboratory of Agriculture \& Agri-Product Safety, College of Agriculture, Yangzhou University, Yangzhou, Jiangsu Province 225009 China (E. Li); Joint International Research Laboratory of Agriculture \& Agri-Product Safety, College of Agriculture, Yangzhou University, Yangzhou Jiangsu Province 225009 China, and also Centre for Nutrition \& Food 
Sciences, QAAFI, The University of Queensland, Brisbane, Qld 4072, Australia

(R.G. Gilbert).

E-mail addresses: en.li@uqconnect.edu.au (E. Li), b.gilbert@uq.edu.au (R.G. Gilbert).

\section{Abstract}

Liver glycogen, a complex branched polymer of glucose, plays a major role in controlling blood-sugar levels. Understanding its molecular structure is important for diabetes, especially since it has been found that this structure is more fragile in diabetic than in healthy mouse liver. However,there are differences in metabolic processes between liver lobes, which would be expected to be reflected in differing glycogen molecular structures. This structure was examined for separated lobe regions in rat livers, usingsize-exclusion chromatography (SEC) and fluorophore-assisted carbohydrate electrophoresis. The results show that the SEC weight distribution of glycogen, and the molecular weight distribution of individual branches (chains), from different lobes are similar.This shows that (a) molecular structural characterization of glycogen from whole-liver biopsy is representative (which is convenient because the commonest animal model for diabetes is the mouse, whose livers are very small), and (b) the fact that molecular structure is conserved (regulated) in different lobes suggests that this structure plays an important role in blood-sugar regulation.

Key words: glycogen;molecular structure;liver; diabetes; GPC 


\section{Introduction}

Glycogen isa complex, highly branched, glucose polymer(containing a small, but functionally important, amount of bound protein[1-3]). Glycogen plays an important role in regulating blood sugar in human and otheranimals [4]. Liver glycogen has three levels of structure: 1$)$ glucosyl monomer units are attached through $\alpha-(1 \rightarrow 4)$ linkages to form linear chains; 2$)$ these chains are joined together via $\alpha-(1 \rightarrow 6)$-linked branch points to form highly branched $\beta$ particles ( 20 nm in diameter); and 3) $\beta$ particles joininto $\alpha$ particles (up to $300 \mathrm{~nm}$ in diameter)[2, 5], which have a composite raspberry- or cauliflower-like appearance under transmission electron microscopy[6]. It has been found [7] that the binding between $\beta$ particles to form $\alpha$ particles is fragile in diabetic liver glycogen, using $d b / d b$ mice as a model for type 2 diabetes. Given that liver glycogen has similar molecular structure in both mice and humans [8], a better understanding of this molecular structure is of significance in the study of human type 2 diabetes.

The liver has multiple distinguishable lobes, and it is possible that the molecular structure of glycogen could be different in different lobes, because there are some differences in the metabolic processes between lobes. Matsubara et al. [9] examined enzyme distributions and reported thatseveral enzymes were distributed heterogeneously in Wistar rat liver lobes, and that the median lobe showed a slightly higher glycogen content. However, this study only used 3 rats and the differences are not statistically significant. Garcia-Moreno et al. [10] found that there are differences in area and number of silver-nucleolar organizer regions (Ag-NORs) in different lobes. As the Ag-NORscan indicatehepatocytic protein synthesis, the results show the existence of a lobular functional heterogeneity in liver. 
The question investigated here is to see if there is a difference in the molecular structure of glycogen in these different lobes, given that there is some metabolic difference between these lobes; this metabolic difference could affect glycogen synthesis and degradation. If there were to be a significant difference, this would give data which could be used to better understand the lobe dependence of this synthesis and degradation, but would also mean that molecular structural data extracted from whole liver is not truly representative. If there were to beno significant difference, this would suggest that this molecular structure is sufficiently important for the well-being of the animal that it is controlled (regulated) in a way that is robust to some metabolic changes (as is seen, for example, in another complex branched polymer with similar bonding to glycogen, amylopectin in plant starch, for which the theoretical reasons for structural robustness are understood [11]). The structural characteristics examined here are the size distribution of the wholemolecule, and the molecular weight distribution of the individual chains (branches), obtained from the whole molecule by enzymatic debranching. Overall glycogen particle morphology is also examined.

Most previous studies on the molecular structure of glycogen have used mice as an animal model. Because mouse liver is small, the glycogen in these studies have perforce been extracted from the whole liver so as to obtain sufficient sample for structural characterization. In order to ascertain the relevance of these mouse studies to humans, it is important to see if there is a difference in glycogen molecular structure in different lobes, which requires having livers which are sufficiently large that enough glycogen for structural characterization can be extracted from different lobes.

This study uses rat livers. Ratshave a similar liver structure to mice but the liver is much larger. Rat liverhas four lobes: left, right, middle and caudate[12]. The 
median lobe can further be groupedinto left and right portions; the right lobe can also be grouped into right superior lobe and right inferior lobe; the caudate lobe can bedivided into the paracaval portion and the Spiegel lobe, whichsplits into two sub-lobes.

Size-exclusion chromatography (SEC, a type of gel-permeation chromatography, GPC) and fluorophore-assisted carbohydrate electrophoresis (FACE)are used here to analyze the molecular structure of extracted glycogen.SEC measures the size distributions of molecularly dispersed molecules as a function of their hydrodynamic radius, $R_{\mathrm{h}}$. With a differential refractive index (DRI) detector, it gives the weight distribution of these molecules as a function of $\log R_{\mathrm{h}}, w\left(\log R_{\mathrm{h}}\right)$. SEC is used here to measure the size distributions of the whole glycogen molecules. The molecular weight distribution of the individual branches (chains - the chain-length distribution, CLD) is measured here by enzymatically debranching the whole molecule, then using FACE to measure the CLD. Although FACE currently cannot go above a degree of polymerization (DP, symbol $X$ ) 180 [13], this is sufficient for the size range of debranched glycogen. FACE directly gives the number distribution of DP following debranching, denoted $N_{\mathrm{de}}(X)$, and gives baseline resolution between DPs in the range of interest. The morphology of the glycogen molecules is examined in this study using transmission electron microscopy (TEM).

\section{Experimental}

\subsection{Animals}

Male Wistar rats (13 weeks)were purchased from the Hubei Provincial Centrefor Food and DrugSafety.Rats were housed in a standard specific pathogen-free(SPF) animal room with standard cages.The temperature was 
controlled at $22 \pm 1^{\circ} \mathrm{C}$ and a 12 -h dark/light cycle was used, with lights on at 7am and off at $7 \mathrm{pm}$. Animals had ad libitum access to water and standard chow (6\% kcal

from fat, 14.3 $\mathrm{MJ} \mathrm{kg}^{-1}$, Hubei Provincial Centre for Disease Control and Prevention). All animal experiments were approved by the Huazhong University of Science and Technology Tongji Medical College Animal Care and Ethics Committee.

\subsection{Excision of liver}

Rats were divided into two groups. Those in Group 1 were sacrificed in the morning, between 9 and 10am, andthose in Group 2were sacrificed in the afternoon between 2to 3pm. These two times are respectivelyatthe beginningand end of the degradation phase in glycogen synthesis[14]. Rats were anaesthetized with sodium pentobarbitone (150 mg/kg intraperitoneal) and their livers were rapidly excised and divided into six parts according to the lobe sections given in Fig. 1.Each lobe was snap-frozen in liquid nitrogen and stored at $-80^{\circ} \mathrm{C}$. 


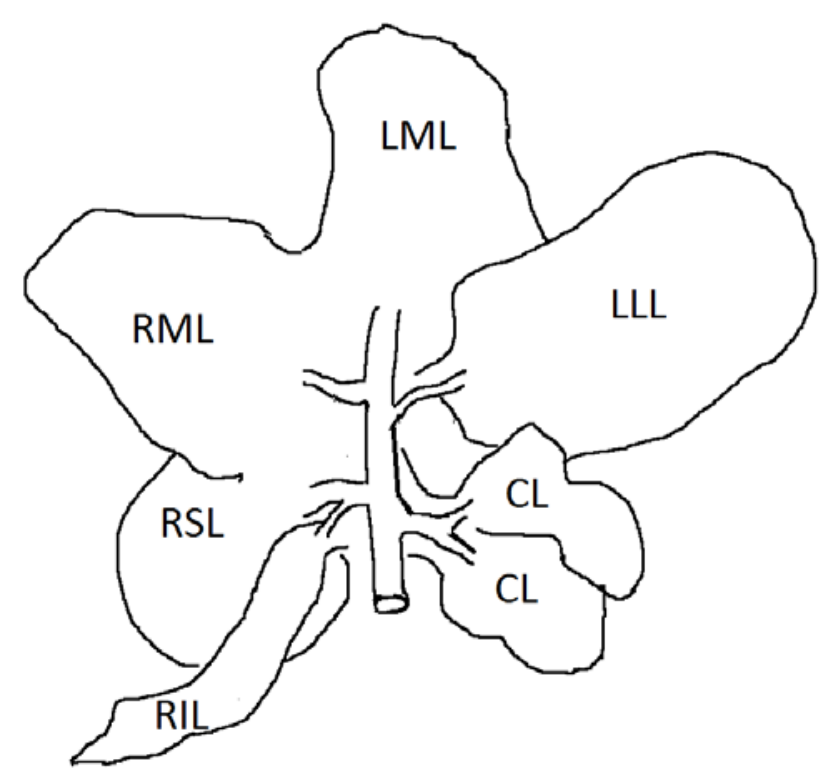

Figure 1. Six lobes of rat liver: left portion of middle lobe (LML), right portion of middle lobe (RML), right superior lobe (RSL), right inferior lobe (RIL), caudate lobe (CL) and left lateral lobe (LLL).

\subsection{Purification and extraction of glycogen}

Glycogen was extractedfollowing a previous procedure[15]. Approximately $1.5 \mathrm{~g}$ of rat liver was homogenized in $25 \mathrm{~mL}$ of glycogen isolation buffer(50 mM Tris, $\mathrm{pH}$ 8, $150 \mathrm{mM} \mathrm{NaCl}, 2 \mathrm{mM}$ EDTA, $50 \mathrm{mM} \mathrm{NaF}$ and $5 \mathrm{mM}$ sodium pyrophosphate). $400 \mu \mathrm{L}$ of this homogenate was reserved for glycogen content assay. The remaining samples were centrifuged at $6000 \mathrm{~g}$ for $10 \mathrm{~min}$ at $4{ }^{\circ} \mathrm{C}$. The supernatants were further centrifuged at $260000 \mathrm{~g}$ for $2 \mathrm{~h}$ at $4{ }^{\circ} \mathrm{C}$. The pellets were then resuspended in glycogen isolation buffer and layered over a20-mL stepwise sucrose gradient (37.5 and $75 \%$ sucrose in deionized water). These samples were then centrifuged at $370000 \mathrm{~g}$ for $2.5 \mathrm{~h}$ at $4{ }^{\circ} \mathrm{C}$. The pellets of glycogen at the bottom of the tube were resuspended in $0.5 \mathrm{~mL}$ of deionized water. Samples were mixed 
with four times volume ofabsolute ethanol to precipitate the glycogen. The samples were centrifuged at $4000 \mathrm{~g}$ for $10 \mathrm{~min}$ and the pellets were re-dissolved in $1 \mathrm{~mL}$ of deionized water and lyophilized (freeze-dryer; VirTis, BTP-9EL).

\subsection{Glycogen content}

The liver-glycogen content was analyzed using a method reported elsewhere[14, 16]. Liver homogenates from Group 1 (sacrificed in the morning) were used for glycogen content assay. Amyloglucosidasewas used to degrade glycogen to glucose units and then glucose oxidase/peroxidase (GOPOD, Megazyme, Ireland) reagent to quantify the amount of glucose. $20 \mu \mathrm{L}$ of homogenate (from the glycogen extraction) was put in a 1.5mL tube, $5 \mu \mathrm{L}$ of amyloglucosidase (Megazyme), 100 $\mu \mathrm{L}$ of sodium acetate buffer ( $\mathrm{pH} 6$ ) added, then made up to $0.5 \mathrm{~mL}$ with deionized water and incubated in a thermomixer at $50{ }^{\circ} \mathrm{C}$ for $30 \mathrm{~min}$. A control, with everything except amyloglucosidase, was also analyzed. A $300 \mu \mathrm{L}$ aliquot of each sample was then added to $1 \mathrm{~mL}$ of GOPOD and incubated for a further $30 \mathrm{~min}$ at $50{ }^{\circ} \mathrm{C}$ on a thermomixer. The absorbance $(510 \mathrm{~nm})$ of each sample was then analyzed on a UV-61000s UV-vis spectrophotometer (Mapada). The glycogen content was calculated based on a calibration curve (constructed by reacting D-glucose of various concentrations with the same GOPOD reagent). All samples and controls were run in triplicate with the absorbance values averaged.

\subsection{Size-Exclusion Chromatography}

Glycogen from two groups was analyzed using an aqueous SEC system, following a previous method[17],to obtain the size distribution of the whole (undebranched) molecules. Ammonium nitratesolution (50 mM) containing $0.02 \%$ sodium azide (w/w)was used as the mobile phase. Glycogen was dissolvedfor $4 \mathrm{~h}$ at 
$80{ }^{\circ} \mathrm{C}$ in $50 \mathrm{mM}$ ammonium nitrate/0.02 \% sodium azide at $1 \mathrm{mg} / \mathrm{mL}$. Samples were injected into an Agilent 1260 infinity SEC system (Agilent,Santa Clara, CA, USA) using a SUPREMA pre-column, 1000 and 10,000 columns (Polymer Standard Service, Mainz, Germany). The columns were kept at $80{ }^{\circ} \mathrm{C}$ using a column oven; the flow rate was $0.3 \mathrm{~mL} / \mathrm{min}$. A refractive index detector(Optilab UT-rEX, Wyatt, Santa Barbara, CA, USA) was used to determine weight distributions, and multiple-angle laser light scattering detector (DAWN HELEOS-II,Wyatt, Santa Barbara, CA, USA) were used to measure weight-average molecular weight $\left(\bar{M}_{\mathrm{w}}\right)$. Pullulan standards (Polymer Standard Service, Mainz, Germany), with molar

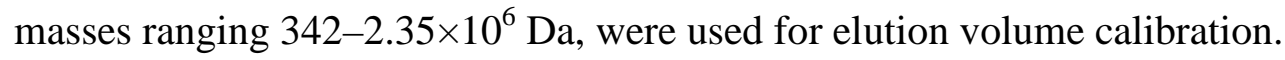

\subsection{Fluorophore-assisted carbohydrate electrophoresis}

Glycogen from Group 1(rats sacrificed in the morning)was analyzed using FACE to give the chain length distribution (CLD) of debranched glycogen from various lobes of livers, following a procedure described elsewhere[13]. Glycogen was firstly debranched and freeze-dried, and then separated with a carbohydrate separation buffer (Beckman-Coulter) in an $\mathrm{N}$-CHOcoated capillary at $25^{\circ} \mathrm{C}$ using a voltage of $30 \mathrm{kV}$.Each sample was analyzed induplicate.

\subsection{TEM}

TEM images of glycogen were obtained using a method similar to that reported elsewhere[18]. Glycogen (from Group 1) was dissolved in 50mM Tris-HCl buffer (pH 7.0) at 1mg/mL. Copper grids (230 mesh) with carbon film were discharged using a Harrick plasma cleaner (PDC-32G-2) before use. The solution was diluted 10 times and applied to the grid for $1 \mathrm{~min}$, and excess sample was drawn off with filter paper. Then the grid was stained with one drop of $2 \%$ uranyl acetate. The grids 
were examined with a Hitachi H-7000 TEM operating at $75 \mathrm{kV}$ using AnalySiS image management software.

\subsection{Statistical analysis}

Analysis of variance (ANOVA) with the general linear model and Tukey's pairwise comparisons were used for statistical analysis in Minitab 16 (Minitab Inc., State College, PA, USA). Significant differences of the mean values were determined at $\mathrm{p}<0.05$.

\section{Results and Discussion}

\subsection{Glycogen content in liver lobes}

Theglycogen contents of homogenate from different lobes in each rat, shown in Table 1, show some apparent differences. However, comparing this content in the same lobe but from different rats as biologicalrepeats, there is nostatistically significant difference ( $\mathrm{p}<0.05, \mathrm{n}=24)$. 
Table 1 The glycogen content of glycogen extracted from various lobes of rat livers (sacrificed in the morning) (means \pm SD) ${ }^{\mathrm{a}}$

\begin{tabular}{ccccccc}
\hline Glycogen content (\%) & LML & LLL & RML & CL & RSL & RIL \\
\hline HR1 & $1.9 \pm 0.2$ & $2.1 \pm 0.1$ & $1.8 \pm 0.2$ & $1.7 \pm 0.1$ & $2.0 \pm 0.1$ & $1.8 \pm 0.2$ \\
HR2 & $2.0 \pm 0.4$ & $2.3 \pm 0.2$ & $2.2 \pm 0.2$ & $2.2 \pm 0.1$ & $2.2 \pm 0.1$ & $1.9 \pm 0.2$ \\
HR3 & $1.2 \pm 0.1$ & $2.1 \pm 0.1$ & $1.8 \pm 0.2$ & $3.5 \pm 0.0$ & $2.2 \pm 0.1$ & $1.9 \pm 0.1$ \\
HR4 & $2.8 \pm 0.3$ & $2.2 \pm 0.2$ & $2.6 \pm 0.5$ & $3.6 \pm 0.2$ & $1.0 \pm 0.0$ & $2.3 \pm 0.2$ \\
HR5 & $2.6 \pm 0.1$ & $1.9 \pm 0.3$ & $2.6 \pm 0.2$ & $2.3 \pm 0.2$ & $2.7 \pm 0.3$ & $2.4 \pm 0.1$ \\
HR6 & $2.4 \pm 0.1$ & $2.2 \pm 0.1$ & $2.3 \pm 0.2$ & $2.0 \pm 0.1$ & $2.5 \pm 0.1$ & $2.2 \pm 0.1$ \\
HR7 & $2.8 \pm 0.1$ & $2.4 \pm 0.3$ & $2.5 \pm 0.3$ & $1.8 \pm 0.1$ & $2.7 \pm 0.2$ & $2.3 \pm 0.1$ \\
HR8 & $3.0 \pm 0.1$ & $2.8 \pm 0.1$ & $2.8 \pm 0.1$ & $2.7 \pm 0.1$ & $2.9 \pm 0.1$ & $2.6 \pm 0.0$ \\
mean & $2.3 \pm 0.6^{\text {a }}$ & $2.2 \pm 0.3^{\text {a }}$ & $2.3 \pm 0.4^{\text {a }}$ & $2.5 \pm 0.7^{\text {a }}$ & $2.3 \pm 0.6^{\text {a }}$ & $2.2 \pm 0.3^{\text {a }}$ \\
\hline
\end{tabular}

a Mean \pm standard deviation is calculated from duplicate measurements.

Values with different letters in the same row are significantly different at $\mathrm{p}<$ 0.05 .

Molecular size distributions of glycogen from various lobes

For healthy rats sacrificed in the morning, the SEC weight distributions of liver glycogen from different lobes, $w\left(\log R_{\mathrm{h}}\right)$, are shown in Figs.2 and 3. The data are normalized to the height of the highest peak.A main peak with a distinct shoulder peak was observed for most of the samples. The main peak, withmaximum at $R_{\mathrm{h}} \sim 40$ nm,comprises $\alpha$ particles (Table 2 and Fig 2), and the shoulder at $R_{\mathrm{h}}=$ 20 25nmcomprises $\beta$ particles. The molecular structure of glycogen from different lobes in each individual rat show apparent differences in the size distribution, especially in the ratio of $\alpha$ and $\beta$ particles. However, when comparing glycogen from the same lobe but from different rats as biological repeats, the molecular size distributions of $\alpha$ particlesfrom different lobes of the same rat aresimilar (Table 2).While thereare differencesin thefraction of $\beta$ particlesindifferent lobes (quantified 
as ratio of the areas under the $w\left(\log R_{\mathrm{h}}\right)$ curvesover $1-25 \mathrm{~nm}$ for $\beta$ particles and 1 100nm for all particles),the differences are small andnotstatistically significantly different.The $\bar{M}_{\mathrm{w}}$ values of glycogen from different lobes are also notstatistically significantly different (Table 2). For the samples obtained in the afternoon, the main peak comprises $\alpha$ particles with maxima at $R_{\mathrm{h}} \sim 37 \mathrm{~nm}$ (Fig.3), where the regions separating $\alpha$ and $\beta$ particles are slightly different fromthose in the morning. Some of these samples show a less obvious $\beta$-particle peak at $\sim 20 \mathrm{~nm}$, corresponding to degradation of $\beta$ particles during the day. The shift of the peakposition of $\alpha$ particles is probably because the samples were analyzed at slightly different times. 

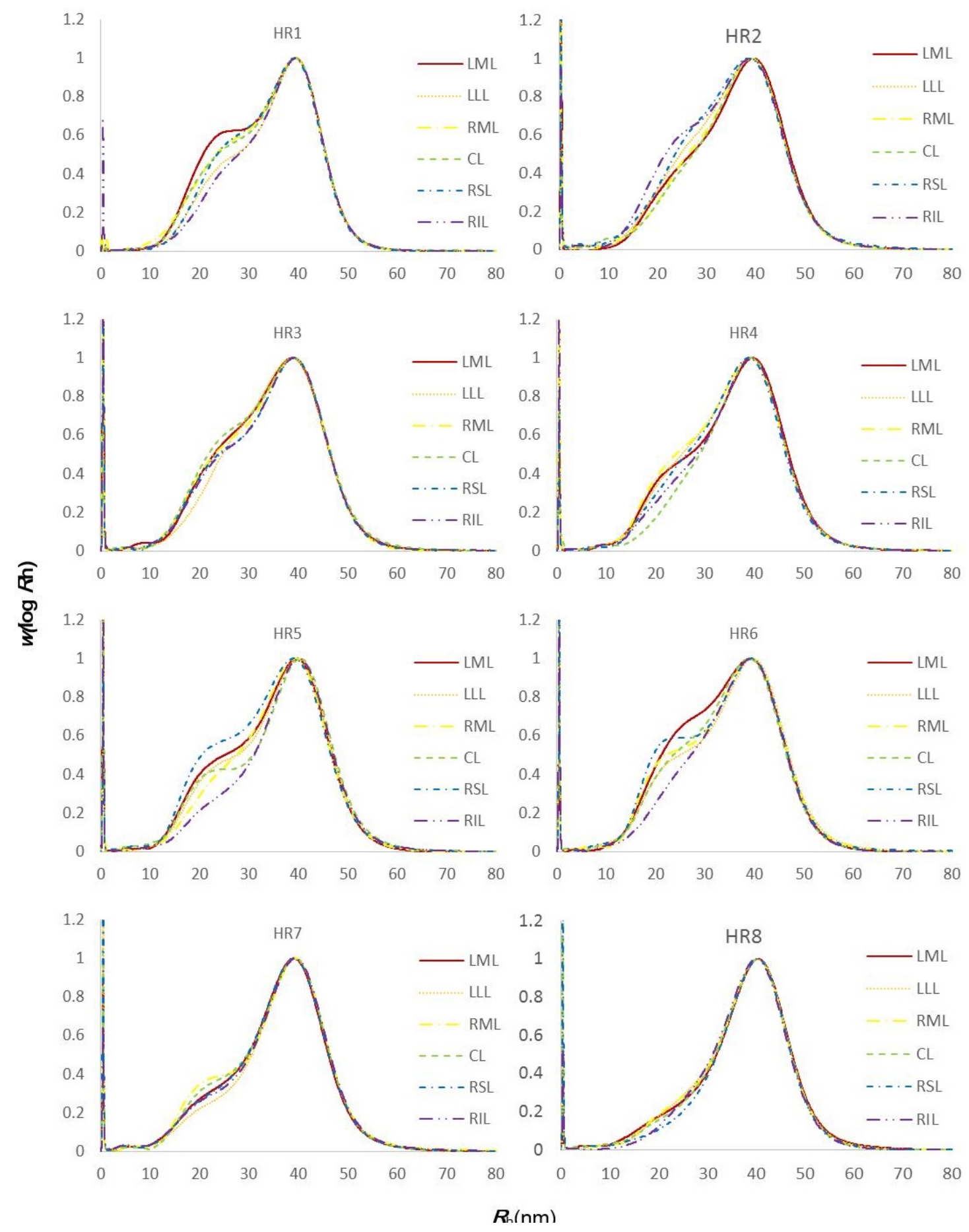

Figure 2. SEC weight distribution, $w\left(\log R_{\mathrm{h}}\right)$, of whole (undebranched) glycogen extracted from different lobes of rat livers; these rats were sacrificed in the morning. Most of the glycogen comprised $\alpha$ particles. Normalized to have the same maximum height. 
Table 2 Whole molecules: $\alpha$-particlepeak positions, weight-average molecular weight. Debranched molecules: slopes of approximately linear regions (from the ranges indicated) in $\log N_{\mathrm{de}}(X)$. For glycogen extracted from differentrat-liver lobes (samples from rats sacrificed in the morning) $(\text { mean } \pm \text { SD) })^{\mathbf{a}}$

\begin{tabular}{|c|c|c|c|c|}
\hline & $\begin{array}{c}\alpha \text {-particle peak } \\
\text { maximum positions } \\
(\mathrm{nm})\end{array}$ & $\begin{array}{l}\text { proportion of } \beta \\
\text { particles }\left(R_{\mathrm{h}}\right. \\
<25 \mathrm{~nm})(\%)\end{array}$ & $\begin{array}{c}\bar{M}_{\mathrm{w}} \\
/ 10^{8} \mathrm{Da}\end{array}$ & $\begin{array}{c}\text { slope of } \\
\log N_{\mathrm{de}}(X) \\
(20 \leq X \leq 40)\end{array}$ \\
\hline LML & $39.5 \pm 0.5^{\mathrm{a}}$ & $17.0 \pm 0^{\mathrm{a}}$ & $7.5 \pm 0.9^{\mathrm{a}}$ & $0.2 \pm 0^{\mathrm{a}}$ \\
\hline LLL & $39.4 \pm 0.5^{\mathrm{a}}$ & $15.4 \pm 0^{\mathrm{a}}$ & $7.6 \pm 0.6^{\mathrm{a}}$ & $0.2 \pm 0^{\mathrm{a}}$ \\
\hline RML & $39.4 \pm 0.4^{\mathrm{a}}$ & $16.8 \pm 0^{\mathrm{a}}$ & $7.5 \pm 0.8^{a}$ & $0.2 \pm 0^{\mathrm{a}}$ \\
\hline CL & $39.5 \pm 0.6^{\mathrm{a}}$ & $15.9 \pm 0^{\mathrm{a}}$ & $8.2 \pm 3.5^{\mathrm{a}}$ & $0.2 \pm 0^{\mathrm{a}}$ \\
\hline RSL & $39.2 \pm 0.5^{\mathrm{a}}$ & $16.8 \pm 0^{\mathrm{a}}$ & $6.5 \pm 2.1^{\mathrm{a}}$ & $0.2 \pm 0^{\mathrm{a}}$ \\
\hline RIL & $39.5 \pm 0.4^{\mathrm{a}}$ & $14.3 \pm 0^{\mathrm{a}}$ & $6.7 \pm 2.2^{\mathrm{a}}$ & $0.2 \pm 0^{\mathrm{a}}$ \\
\hline
\end{tabular}

${ }^{\mathrm{a}}$ Mean \pm standard deviation is calculated from duplicate measurements. Values with different letters in the same row are significantly different at $\mathrm{p}<$ 0.05 . 

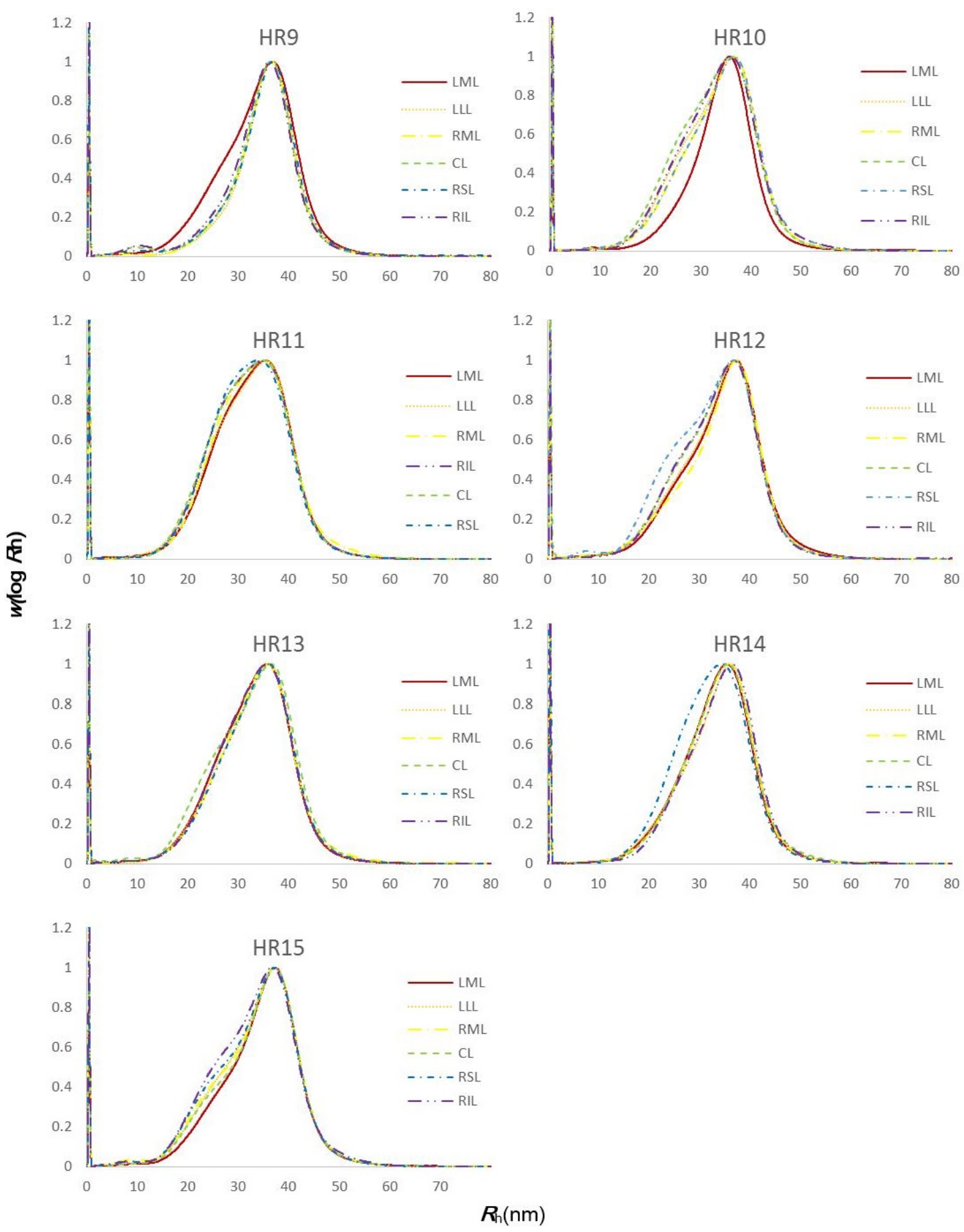

Figure 3. SEC weight distribution, w( $\left.\log R_{\mathrm{h}}\right)$ (normalized to maximum heights), of whole (undebranched) glycogen extracted from different lobes of rat livers; rats sacrificed in the afternoon. Glycogen mainly comprised $\alpha$ particles in these samples. 


\subsection{CLDs of glycogen from different lobes}

Glycogen from different lobesof liver havemeasurable CLDs up to DP 50(Fig 4); above this DP, there is so little glycogen that the signal:noise ratio is very low. These CLDs were quantified by finding theaverage slopesfrom the approximately linear regions in $\log N_{\mathrm{de}}(X)$ inthe range $20 \leq X \leq 40$, using the method of Deng et al. [19].In some of the rats, theCLDsof glycogen from different lobes showsmall apparent differences,but when comparing samples from the same lobe but different rats as biological repeats, the differences are not statisticallysignificant (Table2). 

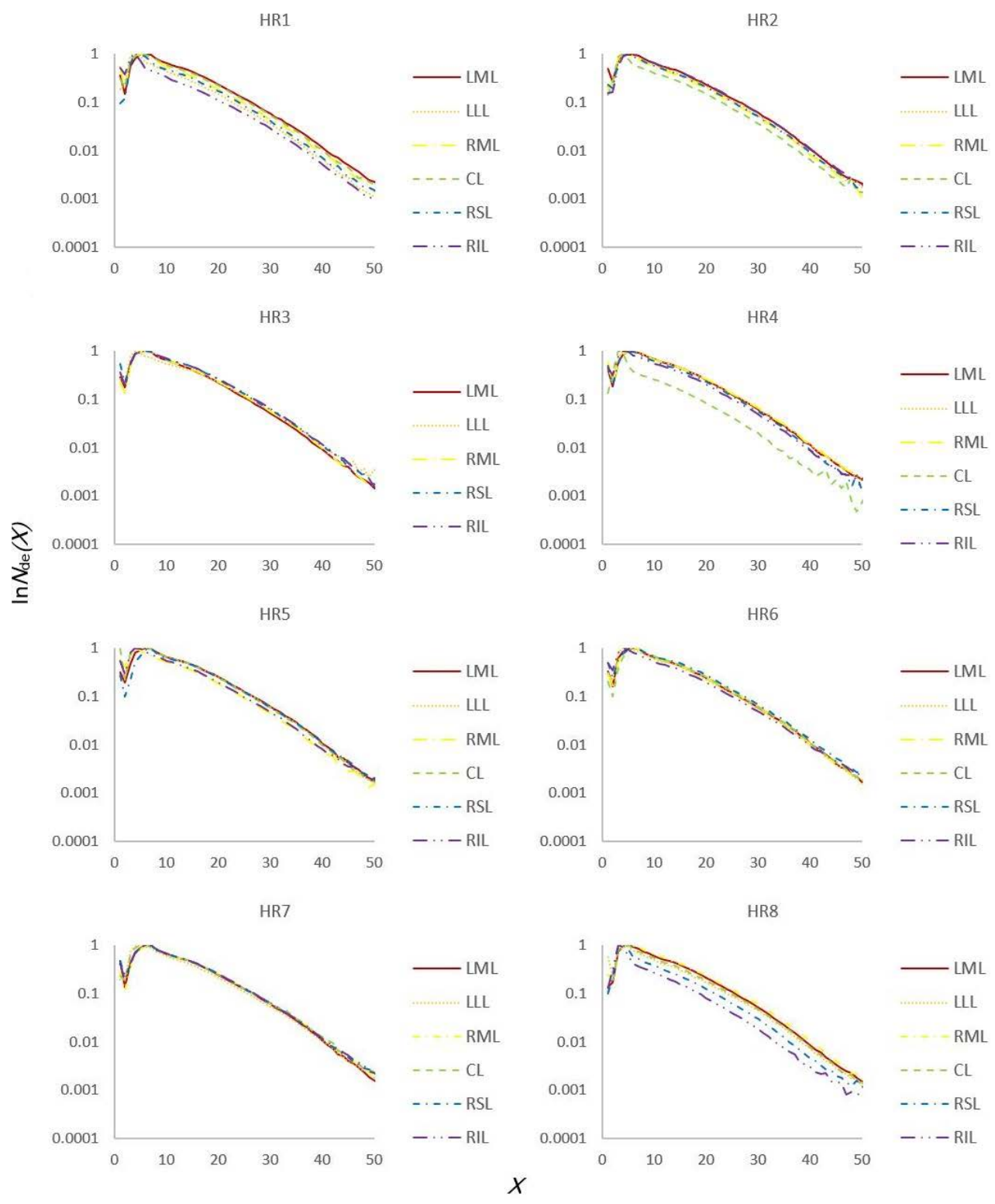

Figure 4. Number CLDs, $N_{\mathrm{de}}(X)$, plotted with a logarithmic $\mathrm{Y}$ axis, of glycogen from various lobes characterized using FACE. All distributions were normalized to the global maximum. Although the FACE data comprise points for individual DPs, they arepresented as continuous lines for visual ease. 


\subsection{Morphology of glycogen molecules}

Representative TEM micrographs of glycogen show large amounts of $\alpha$ particles, and few $\beta$ particles (Fig 5). The $\beta$ particles have diameters around $30 \mathrm{~nm}$. The $\alpha$ particles have a composite, cauliflower-like, appearance with diameters 80 250nm. Particle sizes inTEMare much larger than those inferred usingSEC, for which "size" is hydrodynamic radius. Now, $R_{\mathrm{h}}$ is proportional to cube root of the product of the weight-average intrinsic viscosity and the number-average molecular weight; it has the dimensions of length butis not an easily interpreted physical "size" in the way that the radius of gyration is. Moreover, for a nonglobular polymer like glycogen, the sizes from TEM and SEC can only be compared semiquantitatively[20]. In addition, the samples were under different conditions as the solvent was removed in TEM, which would cause some size change.

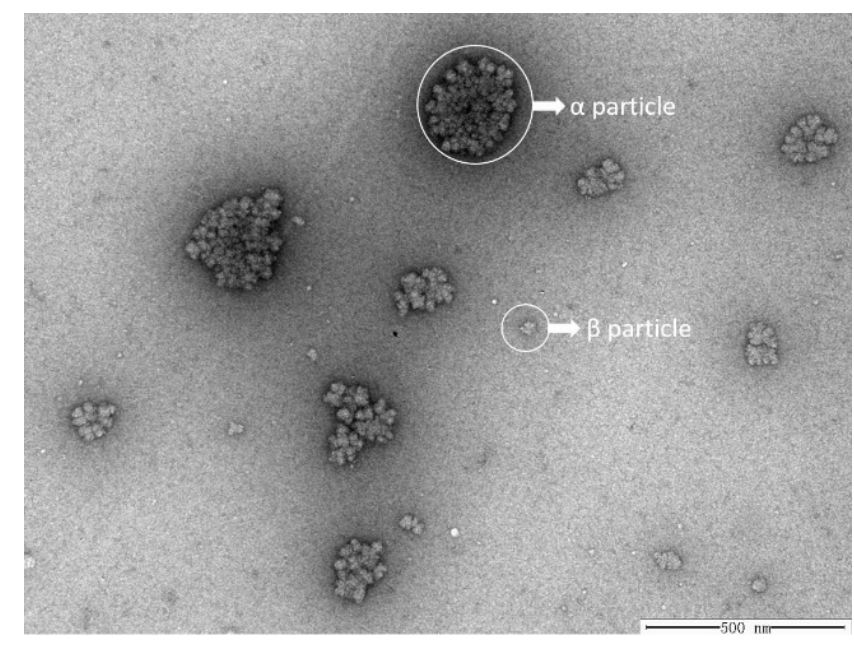

Figure 5. Typical TEM micrograph of glycogen, showingfar more $\alpha$ than $\beta$ particles. The cauliflower-like composite appearance of $\alpha$ particles is very apparent.

SEC and TEM results show that rat glycogen taken at the times used here mainly consisted of many $\alpha$ particles and smaller proportions of $\beta$ particles, as has been seen elsewhere, e.g.[14]. The SEC and FACE results show that glycogen 
samples from different lobes of liver have similar molecular structures, meaning that glycogen extracted from part of liver is representative of the whole.The SEC resultsshowsmall(particularlyrelative amounts of $\beta$ and $\alpha$ particles)and not statistically significant differences for glycogen from different liver lobes. The same holds for the slopes from the FACE results; for most rats, glycogen from different lobes show very similar CLDs. In some rats, there are a few glycogen samples from different lobes that have small differences. However, the slopes of the linear regions in log $N_{\mathrm{de}}(X)$ over the range $20 \leq X \leq 40$ are similar. These slopes yield the ratio of the rates of chain stoppage to growth [19, 21], and thus different lobes have similarglycogen growth kinetics.

\section{Conclusions}

Glycogen from different liver lobes of rats have very similar molecular structures, both for the molecular weight distribution (CLD) of individual chains (branches) and the $\bar{M}_{\mathrm{w}}$ 's and size distributions of the whole molecules. The same similarity is seen in rats sacrificed at different times of the day, when the molecular structures vary with time of day but not with the lobe. Since the variation is small and random within each rat, itmeans that structural characterization of glycogen from any part of the liver isquantitatively representative of that from the whole liver.

This similarity has biological implications. The different lobes have significant differences in metabolic processes, but the liver glycogen molecular structure, which is controlled by metabolic processes, does not show a lobe dependence. The biological function of liver glycogen is essentially controlling blood-sugar storage and release (processes which are compromised in diabetes). This "negative”result of 
lobe structural similarity implies that this molecular structure is biologically regulated, and thus must have a significant role in this biological function, which is to maintain blood-sugar level. The ramifications of this discovery is an area for future research which will be of value to diabetes prevention and management.

\section{Acknowledgements}

The authors thank Mr Shiqing Zhou for technical assistance with SEC and FACE measurements, Ms Prudence O Powell for technical assistance with TEM, andthank the Core Facility and Technical Support, Wuhan Institute of Virology, for the help in TEM. The support of the 1000-Talents Program of the Chinese State Administration of the Foreign Experts Bureau is gratefully acknowledged.

\section{References}

[1] F. Meyer, L. Heilmeyer, R.H. Haschke, E.H. Fischer, Control of phosphorylase activity in a muscle glycogen particle. 1. Isolation and characterization of protein-glycogen complex, Journal of Biological Chemistry 245(24) (1970) 6642-8.

[2] K.K. Rybicka, Glycosomes - The organelles of glycogen metabolism, Tissue Cell 28(3) (1996) 253-265.

[3] D. Stapleton, C. Nelson, K. Parsawar, D. McClain, R. Gilbert-Wilson, E. Barker, B. Rudd, K. Brown, W. Hendrix, P. O'Donnell, G. Parker, Analysis of hepatic glycogen-associated proteins, Proteomics 10(12) (2010) 2320-9.

[4] E.A. Newsholme, C. Start, Regulation of metabolism, Wiley, New York, 1974.

[5] T. Takeuchi, T. Iwamasa, H. Miyayama, Ultrafine structure of glycogen macromolecules in mammalian-tissues, J. Electron Microsc. 27(1) (1978) 31-38.

[6] P. Drochmans, Study under the electron microscope of negative colourings of particulate glycogen, J. Ultrastructural Res. 6 (1962) 141-63. 
[7] B. Deng, M.A. Sullivan, J. Li, X. Tan, C. Zhu, B.L. Schulz, R.G. Gilbert, Molecular structure of glycogen in diabetic liver, Glycoconjugate Journal 32(3-4) (2015) 113-8.

[8] B. Deng, M.A. Sullivan, C. Chen, J. Li, P.O. Powell, Z. Hu, R.G. Gilbert, Molecular structure of human- and mouse-liver glycogen, PLoS One 11(3) (2016) e0150540.

[9] T. Matsubara, A. Touchi, A. Ogawa, Heterogeneous distribution of the cytochrome P-450 monooxygenase system in rat liver lobes, Jpn J Pharmacol 32(6) (1982) 999-1011.

[10] L. Garcia-Moreno, G. Vallejo, J.L. Arias, M.A. Aller, L. Lorente, J. Arias, Behaviour of nucleolar organizer regions in the different Wistar rat liver lobes, Lab Anim 28(1) (1994) 50-4.

[11] A.C. Wu, R.G. Gilbert, Molecular weight distributions of starch branches reveal genetic constraints on biosynthesis, Biomacromolecules 11(12) (2010) 3539-47.

[12] K. Kogure, M. Ishizaki, M. Nemoto, H. Kuwano, M. Makuuchi, A comparative study of the anatomy of rat and human livers, J Hepatobiliary Pancreat Surg 6(2) (1999) 171-5.

[13] A.C. Wu, E. Li, R.G. Gilbert, Exploring extraction/dissolution procedures for analysis of starch chain-length distributions, Carbohydrate Polymers 114(1) (2014) 36-42.

[14] M.A. Sullivan, S.T.N. Aroney, S. Li, F.J. Warren, L. Joo, K.S. Mak, D.I. Stapleton, K.S. Bell-Anderson, R.G. Gilbert, Changes in glycogen structure over feeding cycle sheds new light on blood-glucose control, Biomacromolecules 15(2) (2014) 660-5.

[15] M.A. Sullivan, S. Li, S.T. Aroney, B. Deng, C. Li, E. Roura, B.L. Schulz, B.E. Harcourt, J.M. Forbes, R.G. Gilbert, A rapid extraction method for glycogen from formalin-fixed liver, Carbohydrate Polymers 118(1) (2015) 9-15.

[16] K.L. Roehrig, J.B. Allred, ]Direct Enzymatic Procedure for the Determination of Liver Glycogen, Anal. Biochem. 58 (1974) 414-21.

[17] M.A. Sullivan, P.O. Powell, T. Witt, F. Vilaplana, E. Roura, R.G. Gilbert, Improving size-exclusion chromatography for glycogen, J. Chromatography A 1332(1) (2014) 21-29.

[18] P.O. Powell, M.A. Sullivan, M.C. Sweedman, D.I. Stapleton, J. Hasjim, R.G. Gilbert, Extraction, isolation and characterisation of phytoglycogen from su-1 maize leaves and grain, Carbohydrate Polymers 101(1) (2014) 423-31.

[19] B. Deng, M.A. Sullivan, A.C. Wu, J. Li, C. Chen, R.G. Gilbert, The mechanism for stopping chain and total-molecule growth in complex branched polymers, exemplified by glycogen, Biomacromolecules 16(6) (2015) 1870-2. 
[20] M.A. Sullivan, F. Vilaplana, R.A. Cave, D.I. Stapleton, A.A. Gray-Weale, R.G. Gilbert, Nature of alpha and beta Particles in Glycogen Using Molecular Size Distributions, Biomacromolecules 11(4) (2010) 1094-1100.

[21] P.A. Clay, R.G. Gilbert, G.T. Russell, Molecular weight distributions in free-radical polymerizations. 2. Low-conversion bulk polymerizations., Macromolecules 30 (1997) 1935-46. 